Service social

Compte rendu de : «L'intervention sociale auprès des groupes " par Daniel Turcotte et Jocelyn Lindsay avec la collaboration d'Isabelle Côté et de Geneviève Lamonde.

\title{
Monique Bourget
}

Volume 49, numéro 1, 2002

URI : https://id.erudit.org/iderudit/006880ar

DOI : https://doi.org/10.7202/006880ar

Aller au sommaire du numéro

Éditeur(s)

École de service social de l'Université Laval

ISSN

1708-1734 (numérique)

Découvrir la revue

Citer ce compte rendu

Bourget, M. (2002). Compte rendu de [Compte rendu de : « L'intervention sociale auprès des groupes " par Daniel Turcotte et Jocelyn Lindsay avec la collaboration d'Isabelle Côté et de Geneviève Lamonde.] Service social, 49(1), 1-4. https://doi.org/10.7202/006880ar d'utilisation que vous pouvez consulter en ligne.

https://apropos.erudit.org/fr/usagers/politique-dutilisation/ 


\section{Compte rendu de: "L'intervention sociale au- près des groupes ॥ par Daniel Turcotte et Jocelyn Lindsay avec la collaboration d'Isabelle Côté et de Geneviève Lamonde.}

MONIQUE BOURGET, t.s. Université du Québec en Outaouais

L'intervention sociale auprès des groupes semble revenir en force dans plusieurs secteurs d'activité du travail social, comme le signalent les auteurs. Le recours fréquent aux réseaux naturels et le potentiel d'entraide que représentent les groupes en sont l'explication. Par ailleurs, les écrits en français sur le sujet sont rarissimes et l'héritage de Simone Paré, bien qu'intéressant, date déjà de quelques décennies. Voilà ce qui en fait un ouvrage qui était attendu et qui répond à des besoins évidents.

Le volume est une introduction au travail social auprès des groupes et il comporte huit chapitres. Le premier situe ce type de travail dans son contexte historique. II traite également des valeurs, des objectifs et des connaissances théoriques issues de plusieurs disciplines et qui facilitent l'observation et l'analyse des comportements au sein des groupes et des pratiques diverses. L'objet du chapitre 2 est de savoir comment analyser et comprendre un groupe (but, normes, leadership). On discerne une préoccupation pédagogique des auteurs à transmettre une information utile et bien structurée. Le groupe est un système vivant qui traverse divers stades de développement. Dans le chapitre 3, les auteurs s'attachent au rôle de l'intervenant, particulièrement important dans les prises de décisions en groupe et dans la gestion des conflits ou des difficultés. Les autres chapitres précisent et décortiquent les phases du processus d'intervention. Plus particulièrement, les chapitres 4,5 et 6 sont consacrés à ces différentes phases, aux stades de développement du groupe ainsi qu'aux rôles et 
aux tâches de l'intervenant, lesquels évoluent parallèlement à la progression du groupe. Le chapitre 7 s'attache à répondre à la question suivante: Comment et pourquoi évaluer l'évolution du groupe et mieux suivre le processus? Par ailleurs, le chapitre 8 répondra aux questions des personnes intéressées à la coanimation. On y traite tour à tour des avantages que les membres, les intervenants et l'organisation peuvent en retirer ainsi que les limites qui l'affectent et les éléments à prendre en considération à ce sujet. En conclusion, les auteurs orientent les lecteurs vers des ouvrages spécialisés, susceptibles de répondre à des besoins plus précis, associés notamment à l'enseignement, à l'évaluation et aux questions concernant l'éthique.

La présentation bien structurée de cet ouvrage de type universitaire le rend facile d'accès. Présenté comme introduction au champ d'action qu'est l'intervention auprès des groupes, il remplit tout à fait sa mission. Les nombreux tableaux aident à la compréhension du texte. Les résumés et la bibliographie complémentaire à la fin de chaque chapitre sont autant de points de repère à la fois pour les étudiants intéressés à revoir rapidement la matière et pour les intervenants plus expérimentés et désireux d'actualiser leurs connaissances sur certaines étapes de l'intervention.

Ce volume peut être d'une grande utilité pour l'enseignement. II peut aisément être de lecture obligatoire pour un cours de méthodologie; les chapitres 1 et 7 seront particulièrement pertinents pour aborder les théories et les pratiques d'intervention auprès des groupes.

Pour les travailleurs sociaux et autres intervenants sociaux intéressés à acquérir une formation en intervention sociale auprès des groupes, cette publication est incontournable.

Les autres intervenants sociaux plus ou moins expérimentés, mais désireux de s'initier à cette méthode d'intervention ou de prendre du recul y trouveront une occasion d'évaluer leurs savoirs et leurs savoir-faire. Le dossier de groupe (pages 186 à196) peut être un outil de travail très pertinent et combien utile pour illustrer les propos tenus en enseignement théorique. En formation pratique, les stagiaires pourront y consigner l'information recueillie, 
traiter de la nature des interventions et du contenu des échanges auxquels ils auront participé. Les praticiens intéressés à documenter leur intervention, à assurer la continuité des services ou à se référer à cet outil de travail pour favoriser la pratique réflexive apprécieront particulièrement cette partie du volume.

Les chercheurs et les étudiants du deuxième cycle trouveront aussi un grand intérêt à consulter cet ouvrage. La phase de travail impliquant la structuration des rencontres, le choix et la pertinence des activités, la gestion des situations conflictuelles, l'importance du dossier comme outil d'apprentissage et instrument de collecte de données sont autant de pistes à explorer en recherche sur l'intervention sociale auprès des groupes.

Puisque ce volume est une introduction au travail social auprès des groupes, il aurait été intéressant d'y trouver quelques exemples concrets puisés dans la pratique. On peut également regretter le peu d'applications transférables à des groupes orientés vers l'action sociale où la tâche et l'engagement du travailleur social sont particulièrement importants, notamment dans le processus décisionnel pour aider le groupe à maximiser son efficacité et à soutenir l'intérêt de ses membres.

En ce qui concerne les applications en matière de coanimation, les auteurs passent sous silence la possible collaboration travailleur social de groupe et organisateur communautaire alors que les compétences de l'un peuvent facilement compléter et enrichir celles de l'autre. À mon avis, les auteurs ont manqué une belle occasion de rapprochement entre la méthode d'intervention auprès des groupes et l'organisation communautaire où elle aurait été utile et pertinente.

Les auteurs auraient pu faire ressortir les avantages de l'aide mutuelle dans une perspective de soutien aux intervenants. Dans un contexte où l'organisation du travail exige beaucoup des individus et permet peu d'échanges et de partage entre les divers types de personnel et la direction, un groupe de soutien ou un groupe de supervision peut être une excellente occasion de mettre en commun des connaissances et des compétences, de briser l'isolement professionnel et de favoriser le changement organisationnel. 
Pour résumer, cet ouvrage rappelle toute l'importance de la formation en intervention auprès des groupes et constitue un outil précieux pour l'enseignement en travail social. Ce volume est plus qu'un livre d'introduction. En s'y référant, les travailleurs sociaux et les intervenants sociaux pourront échanger leurs vues sur leur pratique, élargir leur discussion et nourrir leur réflexion ou même s'en servir pour la formation continue. Ce livre a le grand mérite de répondre à des besoins de formation et de mise à niveau professionnelle et peut aisément constituer un outil de référence pour une pratique réflexive. II s'inscrit dans la tradition de l'École de service social de l'Université Laval qui est de soutenir les pratiques de groupes et de favoriser les échanges entre les intervenants et les chercheurs. Cette contribution est particulière et participe à l'évolution des pratiques en intervention auprès des groupes. Les auteurs seraient bien avisés de poursuivre leur démarche dans la perspective d'une éventuelle étude des groupes restreints ou encore d'une étude plus approfondie sur les modèles d'intervention. On ne peut que leur souhaiter tout le succès possible pour ce premier volume. 